livraisons

d'Histoire

de l'Architecture

\section{Livraisons de l'histoire de l'architecture}

$17 \mid 2009$

Émotions patrimoniales I

\title{
Vie, mort et survie des Bouddhas de Bamiyan
}

(Afghanistan)

Life, Death and Survival of the Buddhas of Bamyam (Afghanistan)

Leben, Tod und Überleben der Buddhasstatuen in Bamiyan (Afganisthan)

\section{Pierre Centlivres}

\section{(2) OpenEdition}

\section{Journals}

Édition électronique

URL : http://journals.openedition.org/lha/200

DOI : 10.4000//ha.200

ISSN : 1960-5994

Éditeur

Association Livraisons d'histoire de l'architecture - LHA

Édition imprimée

Date de publication : 10 juin 2009

Pagination : 13-26

ISSN : $1627-4970$

Référence électronique

Pierre Centlivres, "Vie, mort et survie des Bouddhas de Bamiyan (Afghanistan) », Livraisons de l'histoire de l'architecture [En ligne], 17 | 2009, mis en ligne le 10 juin 2011, consulté le 20 mars 2020. URL:

http://journals.openedition.org//ha/200 ; DOl : https://doi.org/10.4000//ha.200

Ce document a été généré automatiquement le 20 mars 2020.

Tous droits réservés à l'Association LHA 


\title{
Vie, mort et survie des Bouddhas de Bamiyan (Afghanistan)
}

\author{
Life, Death and Survival of the Buddhas of Bamyam (Afghanistan) \\ Leben, Tod und Überleben der Buddhasstatuen in Bamiyan (Afganisthan)
}

\section{Pierre Centlivres}

1 L'histoire des bouddhas de Bamiyan est une histoire de croyance et d'idoles, de deuil et de controverse, mais aussi d'une métamorphose de monuments en chefs d'œuvre posthumes du patrimoine. Je vais tenter de retracer quelques étapes, de leur découverte à leur destruction, et aussi de décrire quelques points de vue sur eux et sur leur destinée.

\section{Vie des bouddhas}

2 En juin 1931, les membres du groupe Pamir de la Croisière jaune arrivaient à Bamiyan dont le site constitue alors un des plus prestigieux chantiers de la DAFA, la délégation archéologique française en Afghanistan. L'archéologue Joseph Hackin, directeur de la DAFA, associé à l'expédition, fit à ses camarades les honneurs du site extraordinaire : deux bouddhas géants sculptés dans une falaise dominant une vallée verdoyante, à une altitude de 2500 mètres. Dans la falaise, les membres de l'expédition pouvaient distinguer des centaines de grottes, plus de sept cents, creusées ou aménagées de main d'homme, dont beaucoup étaient ornées de fresques splendides où l'on pouvait reconnaître des bodhisattvas et des divinités nimbées, témoignant du croisement d'influences indiennes, iraniennes, hellénistiques et centre-asiatiques. Le plus grand des bouddhas avait 55 mètres de haut (ill. 1), le plus petit 38 mètres (ill.2). Leur face, à partir de la lèvre inférieure vers le haut, était absente, comme arasée. 
III. 1 : Le grand bouddha (55 mètres). 10 mai 1964

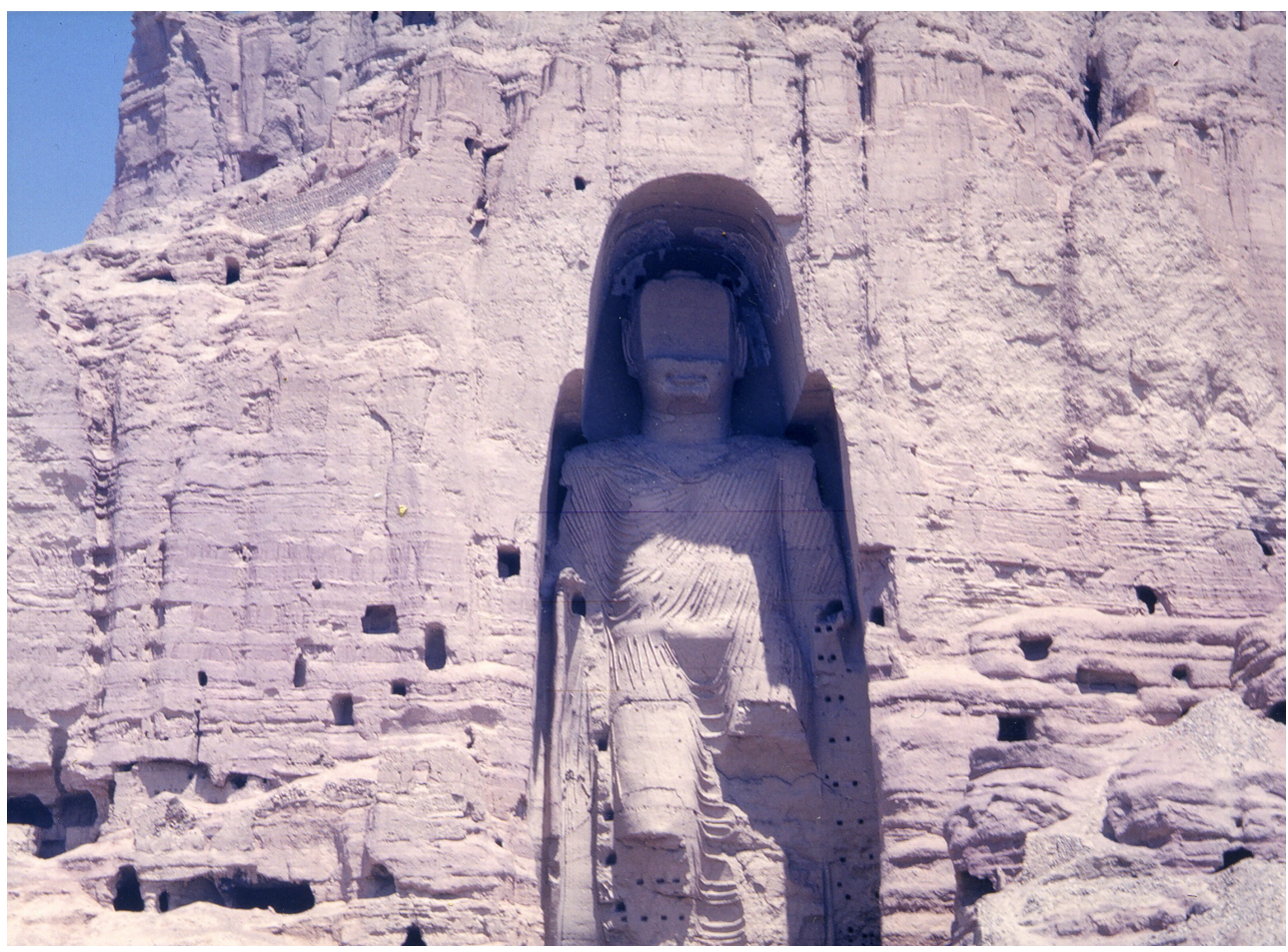

Cl. Pierre Centlivres

III. 2 : Le petit bouddha (38 mètres). 10 mai 1964

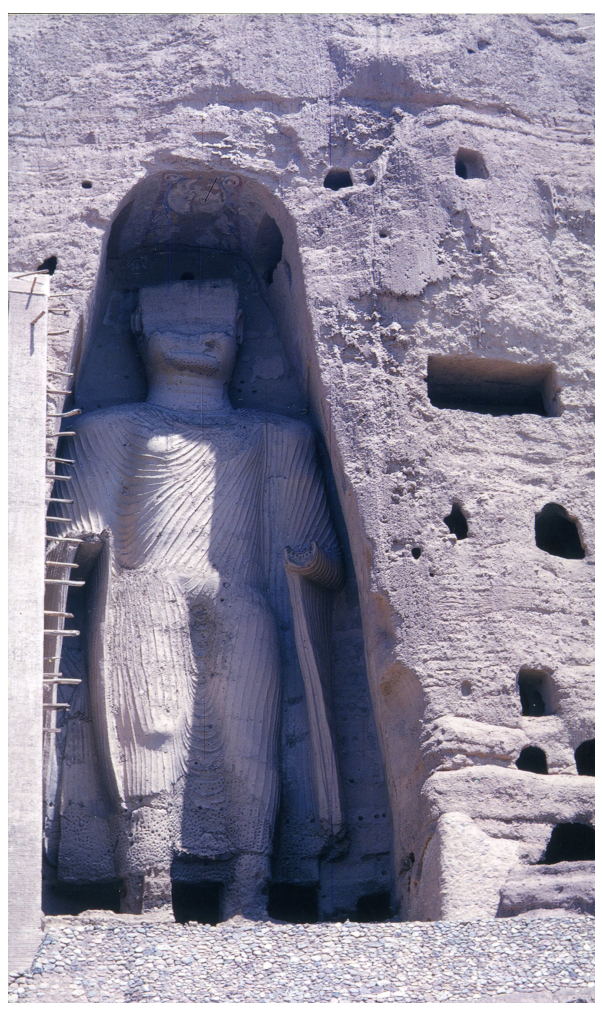

Cl. Pierre Centlivres 
3 Cette visite à Bamiyan, et la contemplation des bouddhas, est un des points forts du récit de Georges Le Fèvre, l'historiographe de la première partie de l'expédition, celle qui relate l'itinéraire du groupe Pamir. Quelques années auparavant, Maurice Fouchet, ministre plénipotentiaire de France auprès du roi Amanullah, écrivait : « Les bouddhas de Bamiyan sont les seules richesses visibles de l'Afghanistan », et il ajoutait: "Le moine philosophe, disciple du Bouddha, a fui devant le mullah fanatique, mais persistent à se dresser les sublimes images de pierre, nées de la foi en un monde supérieur et inconnaissable, par delà l'ignorance nocive et le désir trompeur. Rien, jusqu'à présent, n'a pu détruire ces œuvres géantes, témoins d'un âge où se sont heurtées des conceptions apparemment contradictoires, bien qu'animées du même souffle divin. Sous le soleil implacable dont les rayons ne les ont pas encore vaincues et dissociées, allons leur rendre un hommage pieux. ${ }^{1}$ "

4 C'est ce que font les membres de la mission Citroën, qui, grimpant par d'étroits passages aménagés à l'intérieur de la falaise, se rassemblent sur la tête du grand boudddha, d'où ils ont une vue époustouflante sur la vallée en contrebas et sur la chaîne du Koh-i Baba, qui culmine à plus de 5000 mètres, usurpant en quelques sorte le point de vue du bouddha géant sous sa forme de Seigneur de l'Univers, à la fois protecteur et bienveillant.

5 Redescendu dans la vallée, Hackin s'écrie, tourné vers les faces lisses et aveugles des statues : «L'œuvre des musulmans; leur rage de destruction est une chose incroyable. Les images représentent toujours pour eux les signes d'une redoutable hérésie; les jambes du grand bouddha ont même servi de cibles à l'artillerie de Nader Shah et d'Aurangzeb. ${ }^{2}$ "

Un des thèmes indissociablement lié au deuil des statues est présent de longue date : celui de l'iconoclasme musulman, comme si les talibans, en février-mars 2001, étaient venus à bout de l'obsession destructrice commencée par leurs prédécesseurs, à l'encontre des statues, toujours menaçantes, et enfin abattues.

7 Les archéologues des années trente et bien d'autres avant et après eux étaient donc persuadés que les faces des bouddhas avaient été mutilées par les "fanatiques». Pourtant, dans sa thèse parue dans les années soixante-dix, un archéologue afghan, Zemaryalai Tarzi, faisait remarquer que ces faces, loin d'être sauvagement détruites, avaient été taillées avec soin et ramenées en un plan vertical, avec une rigole à la base. Pourquoi des iconoclastes se seraient-ils donné la peine d'effectuer une taille précise et géométrique de ces visages ? Dans l'hypothèse de Tarzi, les bouddhas de Bamiyan n'ont jamais eu de visage permanent, mais des masques mobiles en bois que l'on changeait selon les cérémonies. Derrière le masque, il y avait le vide.

8 La source principale sur le Bamiyan antique du temps du bouddhisme florissant est un pèlerin chinois, Hiouan Tsang, qui séjourna dans la vallée au cours d'un voyage en Inde à la recherche des manuscrits canoniques du bouddhisme. C'était en 632 de notre ère, année qui est, étrange coïncidence, celle de la mort de Mahomet. Hiouan Tsang décrit la vallée aux mille grottes, aux cent sanctuaires et aux milliers de moines, de prêtres et de pèlerins. Il dépeint les bouddhas géants, le grand dont « le teint d'or est éclatant, et les ornements précieux resplendissants ", et le " petit », qu'il dit être revêtu de plaques en métal brillant. Il signale aussi un immense bouddha en nirvana, un bouddha d'une longueur de mille pieds. Ce bouddha couché fait partie des mythes de Bamiyan, que la légende a métamorphosé en dragon et que nul n'a vu depuis le passage du pèlerin 
chinois. L'archéologue Tarzi, professeur d'archéologie orientale à l'université Marc Bloch, Strasbourg, n'exclut pas cependant de pouvoir le découvrir un jour dans le sol de la vallée : il en est à sa quatrième ou cinquième campagne de fouilles. Les bouddhas datent probablement des $\mathrm{VI}^{\mathrm{e}}$ et $\mathrm{VII}^{\mathrm{e}}$ siècles de notre ère. Leur découverte par les Occidentaux, au début du XIX ${ }^{e}$ siècle, est à inscrire dans le contexte du Great Game, ce combat des ombres, comme disent les Russes, qui opposa par Afghans interposés les deux empires, le russe et le britannique, aux confins de l'Asie centrale et de l'Inde britannique.

9 Les voyageurs européens qui, les premiers, mentionnent dans leurs récits les statues géantes, furent pour la plupart des Britanniques plus ou moins agents secrets, explorateurs et trafiquants. Moorcroft et Trebeck ${ }^{3}$, en route pour le Turkestan afin d'y acheter des chevaux pour l'armée des Indes, passent par Bamiyan en août 1824. Ils mentionnent les deux idoles, comme ils disent, un homme et une femme, selon les propos de leurs guides indigènes. En 1832, cent ans avant le passage de la Croisière jaune, Alexander Burnes ${ }^{4}$ décrit le « couple d'idoles », objets d'un culte révolu (ill. 3).

\section{3 : Les bouddhas de Bamiyan}

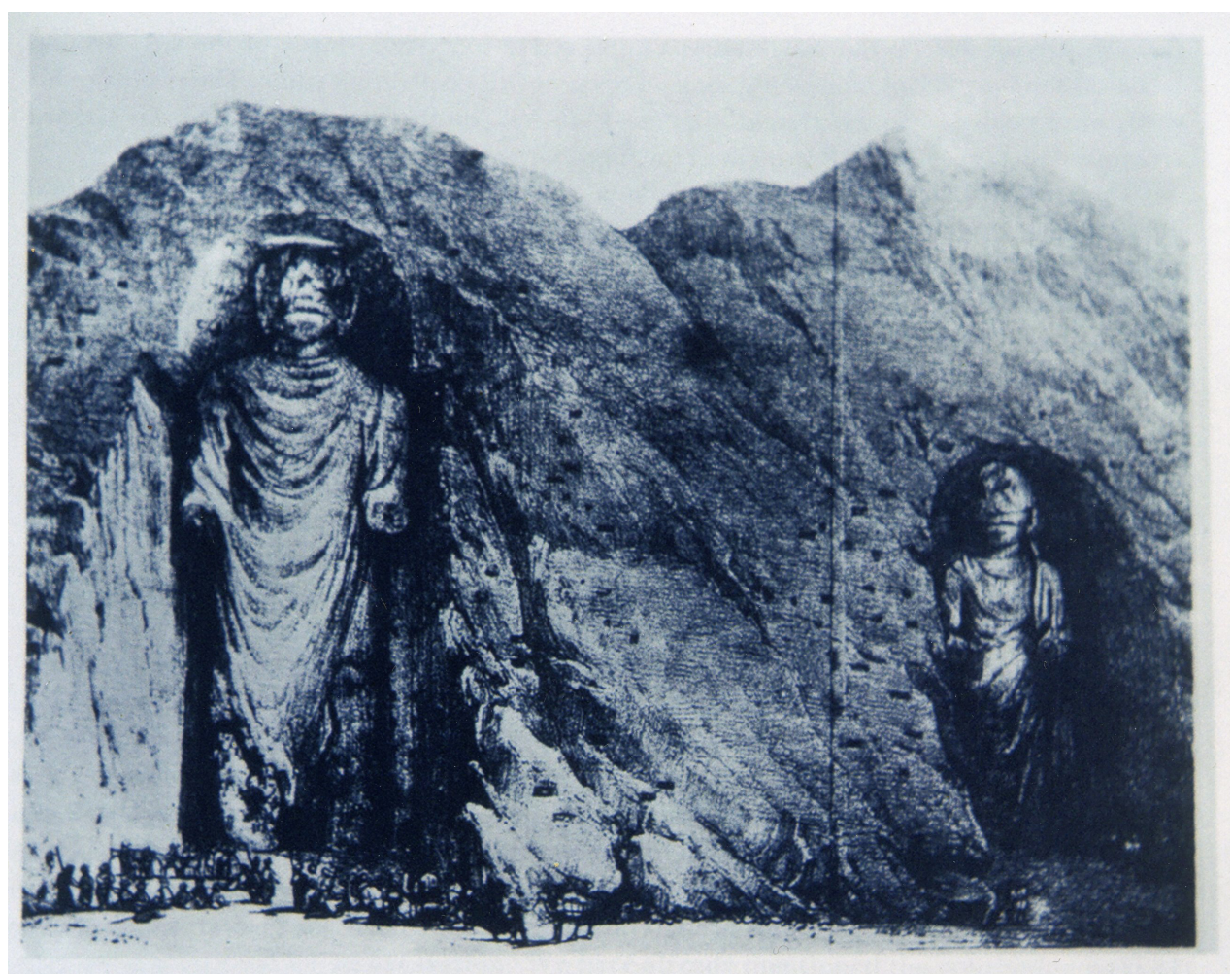

Dessin d'après Alexander Burnes. Alexander Burnes, Travels into Bokhara. 3 vol., Londres, John Murray, 1835, 1835, vol. II, p. 158

10 Mieux, il en laisse un croquis. Curieux dessin! Burnes fait des deux statues des êtres difformes avec une tête où l'on devine un visage grimaçant! Amateur d'antiquité classique, Burnes, un agent de l'Indian Political Service soucieux des menées russes en Asie centrale, polyglotte et déguisé en Afghan, esquisse ce qu'il croit être des divinités païennes; il les trouve inélégantes, asymétriques, disgracieuses même, dignes des sauvages de l'endroit et de leurs croyances primitives. Le dessin est repris en 1848 dans le volume de L'Univers pittoresque consacré à l'Afghanistan, où sont accentués encore les 
traits caricaturaux du Grand bouddha, en esquissant le rictus d'une bouche et les fentes d'une paire d'yeux. Charles Masson, lui, visite Bamiyan en 1835 ; déserteur, trafiquant d'antiquités, espion à la solde de la compagnie des Indes, il est le premier à reconnaître, dans les statues, l'effigie du bouddha, et le premier à les admirer : « Le voyageur, écritil, contemplant les grandes et mystérieuses idoles et la multitude des cavités qui les entourent, ne peut manquer d'être absorbé dans une réflexion et un émerveillement profond..$^{5}$ »

11 Émerveillement et recueillement sont désormais les sentiments éprouvés par les voyageurs, touristes et savants d'Occident, ou du moins presque tous devant les géants de Bamiyan. Le capitaine Maitland en fait un dessin assez exact en $1886^{6}$ (ill.4).

\section{4 : Les bouddhas de Bamiyan}

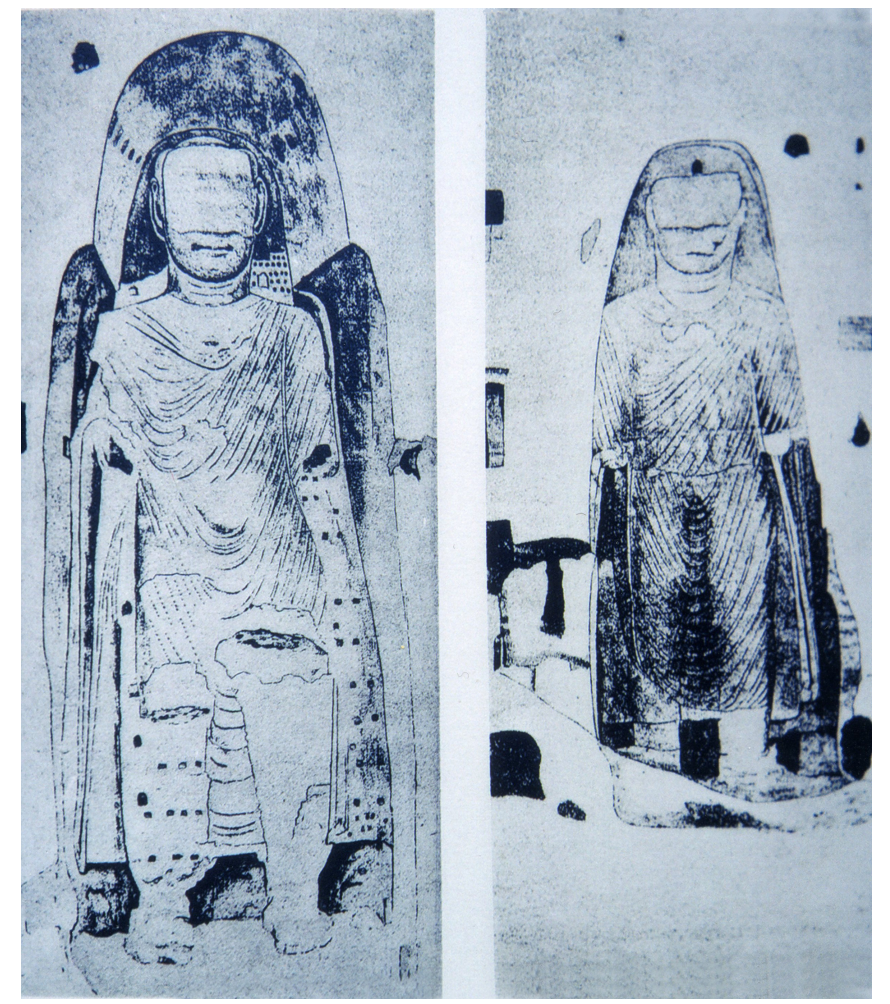

D'après un croquis du capitaine P. J. Maitland, 1886. André Godard, Yeda Godard et Joseph Hackin, Les Antiquités bouddhiques de Bâmiyân. MDAFA II. Paris et Bruxelles, Van Oest, 1928, 113 p., pl. VIII

Ce dessin et celui de Burnes inspirèrent le graphiste auteur de l'image du bouddha, dont l'original est désormais détruit, reproduite au tiers de la taille originale, qui orna en avril 2001 la façade du Centre Pompidou. Curieusement, sur l'image de Beaubourg, on distinguait l'ombre d'un nez, d'une bouche et des yeux, comme si la vacuité de la face qui venait de disparaître à Bamiyan avait quelque chose d'insoutenable pour le public parisien, ou comme s'il fallait humaniser le bouddha au moment où l'on en faisait le deuil.

Du capitaine Maitland au ministre Fouchet, de la Croisière jaune à Ella Maillart qui fit le détour par la haute vallée en 1939, des archéologues et des historiens de l'art aux hippies du Grand Tour des années soixante et soixante-dix, qui s'arrêtent à Bamiyan avant de poursuivre vers Katmandou, la plupart communient dans l'admiration du site et des statues. Une exception pourtant, sorte d'iconoclasme littéraire dû à Robert 
Byron, un voyageur-écrivain-dandy des années trente. Dans The Road to Oxiana, traduit en français sous le titre de Route d'Oxiane, il écrit des deux bouddhas : "Ni l'un ni l'autre n'a de valeur artistique. Mais cela serait encore supportable : l'écœurement naît de la négation de tout sens qu'ils incarnent, du manque de toute fierté au sein de leur monstrueuse masse flaccide. La matière même dans laquelle ils sont taillés est dénuée de beauté, car la falaise n'est pas composée de roche, mais de poudingue. À un certain nombre de moines terrassiers on a donné des pics et dit de copier une hideuse image pseudo-hellénistique venant de l'Inde ou de la Chine. Le résultat n'a même pas la dignité d'un travail de bon ouvrier. ${ }^{7}$ " Malgré Robert Byron et sa description démystificatrice, non exempte d'un snobisme d'enfant gâté, Bamiyan, de 1930 à 1980, était une destination obligée pour les étrangers vivant ou de passage à Kaboul et constitue le plus beau fleuron de l'organisation afghane du tourisme.

Les Afghans justement... Il est difficile de se faire une idée des représentations qu'ils se faisaient des bouddhas. Les habitants de la vallée utilisaient les grottes comme abris et comme greniers à blé, et la fumée de leurs foyers avait recouvert et endommagé les superbes fresques des sanctuaires et des cellules creusées dans le rocher. Tout en étant convaincus que les deux bouddhas géants formaient un couple, ils les nommaient parfois Lât et Manât, les "sublimes Déesses » mentionnées dans le Coran ${ }^{8}$, deux idoles d'un trio de statues des environs de La Mecque que Mahomet avait fait détruire. Les mêmes idoles sont au cœur des versets sataniques et du livre de Salman Rushdie.

Bouddha se dit bud/but dans le monde musulman, mais le terme signifie aussi une idole quelconque, et pas seulement le Bouddha. Il y a donc dans les représentations de beaucoup d'Afghans une association entre les deux grandes statues et des idoles monstrueuses, sorties du Coran. Ces idoles, au nombre de 360 à La Mecque, furent détruites par le Prophète. Dans les années Trente, les postes afghanes émirent un timbre à l'effigie du grand bouddha; mais devant l'opposition des mullahs, il fallut le retirer. Le timbre fut réédité, cette fois-ci sans grande opposition, après la Seconde Guerre mondiale. Pour les habitants du cœur de l'Afghanistan, les bouddhas représentaient donc des présences familières, légèrement sulfureuses, ancrées dans les croyances religieuses comme des survivances des temps préislamiques, et dont les mullahs rappelaient à l'occasion les origines païennes.

\section{Mort des bouddhas}

16 Tout change dans les années quatre-vingt avec le conflit afghan. Le Hazarajat, dont la vallée de Bamiyan est le centre, reste en marge des combats entre troupes gouvernementales et soviétiques, d'une part, et moudjahidin, de l'autre. Après la chute du régime communiste en 1992, le Hazarajat s'assure une quasi-autonomie sous l'égide du parti de l'Unité. Bamiyan devient la capitale de ce que les nationalistes hazaras appellent désormais le Hazaristan, le pays des Hazaras, à partir d'un mythe ethnique, celui d'un peuple hazara homogène, chiite, distinct des autres régions et des autres populations de l'Afghanistan.

Sans revendiquer toutefois une indépendance complète par rapport à Kaboul, les leaders hazaras et le parti de l'Unité mettent sur pied à Bamiyan le gouvernement d'un État quasi souverain avec ministères, parlement et armée. Des liens étroits sont noués avec l'Iran chiite. Pour la première fois depuis la conquête islamique, le site de Bamiyan avec ses grottes et ses statues, qui n'avaient guère, pour les premières, qu'une fonction 
prosaïque d'abri et d'entrepôt, pour les stocks de munitions en particulier, et qui servaient vaguement, pour les secondes, de rappel des temps idolâtres et de divertissements que les conquérants divers prenaient pour cibles, pour la première fois donc depuis mille ans, le site et ces monuments retrouvent une signification emblématique pour le peuple de Bamiyan. Pour les intellectuels hazaras, les gigantesques statues représentent les ancêtres de la population locale, et ce n'est pas un hasard, selon eux, si les conquérants sunnites se sont acharnés contre eux, contre leurs visages en particulier. Les Hazaras d'aujourd'hui seraient donc leurs héritiers ou leurs descendants légitimes. Selon eux, c'est pour porter atteinte à cette légitimité, à cette preuve d'autochtonie que les talibans, doublement adversaires des Hazaras comme sunnites et comme pachtounes, se sont acharnés contre elles. Notons que cette affirmation d'autochtonie, de commune origine des statues et des Hazaras contredit un autre mythe historique cher aux mêmes Hazaras selon laquelle les habitants de la vallée seraient les descendants des guerriers de Gengis Khan.

18 La prise de Bamiyan en septembre 1998 par les talibans permet à ces derniers de réinstaller, pour un temps, une suprématie pachtoune sur les Hazaras, une suprématie des sunnites sur les chiites. La destruction des statues représente certes un attentat contre le patrimoine mondial, mais tout d'abord contre le patrimoine et l'identité hazaras.

19 Voici, en quelques dates et faits, la chronologie des événements menant à la destruction totale des bouddhas. Les talibans s'emparent de Kaboul à la fin du mois de septembre 1996, et de Bamiyan deux années plus tard. Déjà à ce moment, entre 1998 et 2001, des commandants talibans locaux s'attaquent aux bouddhas, mais ils sont désavoués par les autorités centrales. En juillet 1999, le ministère de la culture du régime taliban publie des décrets en faveur de la protection des antiquités et d'autres objets du passé. Mais entre décembre 2000 et janvier 2001, les sanctions contre l'Émirat d'Afghanistan votées par le conseil de sécurité des nations unies entrent en vigueur. Le 14 février 2001, l'opposition hazara reprend Bamiyan qu'elle doit abandonner quelques jours plus tard. Le 26 février, Mullah Omar, l'émir des croyants et la suprême autorité des talibans, promulgue un décret, après consultation d'un collège d'oulémas, ordonnant la destruction des statues et des sanctuaires non islamiques. À cette occasion, Mullah Omar assure cependant que les temples hindous et sikhs d'Afghanistan seront épargnés. Entre février et mars 2001, l'UNESCO, des personnalités culturelles, médiatiques et politiques, ainsi que l'organisation de la conférence islamique se mobilisent et interviennent pour la sauvegarde de l'héritage préislamique d'Afghanistan. Certains États, l'Inde, la Thaïlande, le Sri Lanka, l'Iran même, ainsi que le Metropolitan Museum de New York, offrent de racheter les statues. En vain ! Le 14 mars 2001, les talibans annoncent la destruction complète des bouddhas de Bamiyan et des statues du musée national afghan, ainsi que de celles des autres musées d'Afghanistan. La destruction, disent-ils, a nécessité une semaine d'efforts et de grands moyens pyrotechniques, l'artillerie, des explosifs, entre autres. Mullah Omar ordonne, le 16 mars 2001, le sacrifice de cent vaches dont la viande doit être distribuée aux pauvres, afin d'expier, dit-il, le retard apporté par les musulmans dans la destruction des idoles. Le 19 mars, une séquence filmée de la destruction des bouddhas est montrée par la chaîne de télévision al-Jazira; le 22, les journalistes peuvent constater de visu que le musée de Kaboul est vide, et le 26 mars 2001, les mêmes journalistes sont conviés à Bamiyan pour constater l'anéantissement des deux statues (ill. 5). 


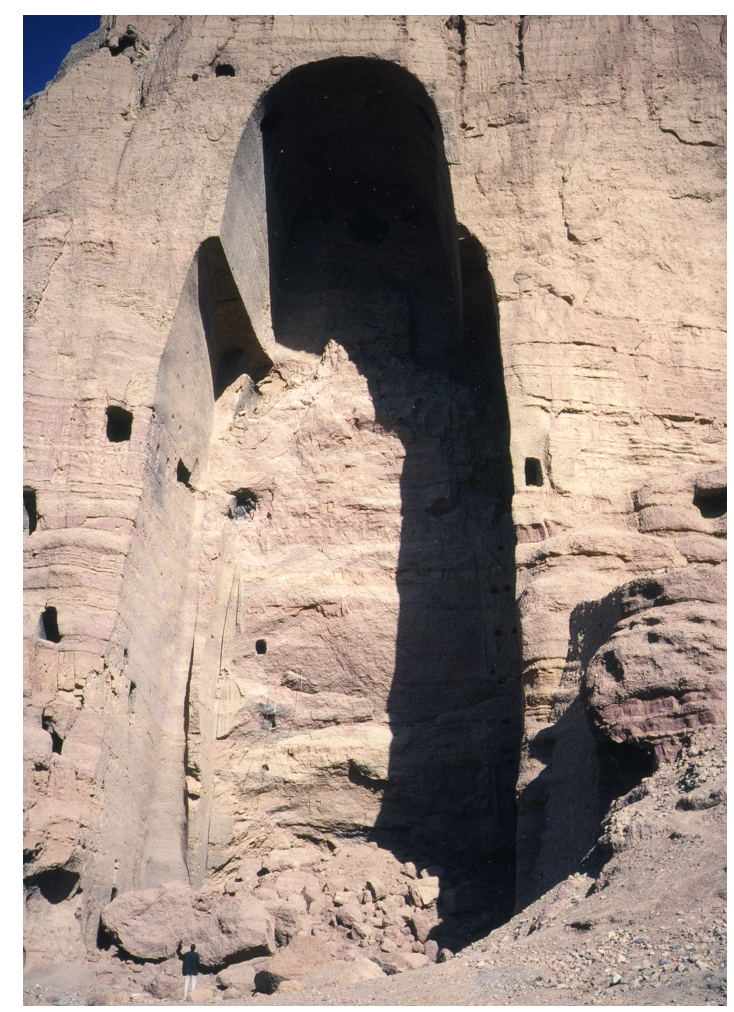

Cl. Pierre Centlivres

20 J'aimerais revenir sur un aspect déjà entrevu de cet extraordinaire happening: la dualité sexuelle ambiguë des statues ou, plus précisément, la connotation féminine d'un des deux monuments. Les médias occidentaux, et les caricaturistes en particulier, ont fait grand usage d'une métaphore, où les hautes figures sans visage sont comparées aux femmes d'Afghanistan sous le châdri. Lors des déprédations d'avant février 2001, précédant la destruction totale, le petit bouddha, censé représenter un être féminin, avait été gravement endommagé ; la tête avait été pulvérisée, et un tir de roquette ouvrit un trou béant à la place du bas-ventre. Il était tentant d'en conclure qu'on avait voulu marquer d'infamie l'offensante présence d'une image féminine, comme si le regard vide de la statue et son sexe de femme supposée faisait encore peur, et qu'il était nécessaire de neutraliser et de déshonorer la statue. Il était tentant également, dans une logique féministe, de voir dans l'anéantissement des Bouddhas, du moins dans l'acharnement contre l'un d'entre eux, une métaphore de l'exclusion des femmes du domaine public et du sort pitoyable qui était le leur sous le régime des talibans.

Dans les années Quatre-vingt, l'UNESCO avait retenu quatre sites afghans comme dignes de faire partie du patrimoine mondial, dont, bien sûr, Bamiyan. Mais à cause du conflit, l'inscription ne put se faire officiellement. Le décret du ministère afghan de la culture de juillet 1999, pris à la demande instante d'un groupe de pression pour le sauvetage du patrimoine culturel et formé de personnalités étrangères ayant des attaches avec l'Afghanistan, semblait garantir la préservation des bouddhas. Ce décret, auquel j'ai déjà fait allusion, affirmait la nécessité de protéger et de conserver le patrimoine culturel de l'Afghanistan, en raison de son ancienneté : il interdisait les fouilles faites par des particuliers, il mentionnait spécifiquement les statues, en 
précisant - à tort - que, depuis l'arrivée de l'islam en Afghanistan, elles n'avaient subi aucune dégradation. Le ministère parlait du "respect » à leur égard et évoquait les revenus futurs du tourisme. Il mentionnait en outre le risque de représailles contre des mosquées dans les pays majoritairement bouddhistes. Enfin, il précisait qu'il n'y avait pas de pratiquants du bouddhisme en Afghanistan et concluait par ces mots: «Le gouvernement des talibans affirme que "Bamiyan ne sera pas détruit, mais au contraire protégé". "Vint le décret du 26 février 2001, qui représentait une véritable volte-face et un désastre pour le patrimoine afghan. Il ordonnait la destruction de toutes les statues et sanctuaires non islamiques d'Afghanistan. "Ces statues » affirmait le décret « ont été et restent des sanctuaires d'infidèles. Ces derniers continuent à adorer et à vénérer ces images et ces statues ». L'ordre de destruction de toutes les statues, celles des musées également, est répété à trois reprises dans le décret.

L'argumentation de la délégation de l'UNESCO présidée par l'ambassadeur Pierre Lafrance auprès des talibans s'appuyait moins sur la notion de patrimoine et sur la charte de l'UNESCO que sur la distinction entre idolâtrie et exemplarité, entre idole et icône et entre respect et culte; ou plutôt, il cherchait à décomposer la notion de patrimoine, en l'occurrence le patrimoine afghan préislamique, en des composantes pouvant servir de base de discussion avec les talibans, telles le respect de la croyance, la connaissance scientifique, la tolérance de la diversité.

Pour Pierre Lafrance, « les statues ne sont pas des objets de culte, mais des rappels de la vertu d'un enseignement, d'une sagesse; les qualifier d'idoles est faire insulte au bouddhisme ». De même, "Les vestiges anciens en général sont devenus des objets de recherche scientifique; or, l'islam respecte le travail des savants de toutes les disciplines » et «les dirigeants musulmans de toutes les époques en Afghanistan ont respecté ces vestiges ». Enfin, d'une façon un peu contradictoire : «Les statues peuvent être l'occasion d'une leçon de foi pour les fidèles, à quelque religion qu'ils appartiennent, ce qui est jugé bénéfique par la tradition islamique. ${ }^{9}$ "

Quant au nouvel argumentaire des talibans, il représente un revirement total par rapport au décret protecteur de 1999, et s'exprime par une sorte de dilemme sophistique qui n'est pas sans rappeler celui qui fut attribué au calife Omar, à propos de l'incendie de la bibliothèque d'Alexandrie. On sait qu'il n'y était pour rien. Voici ce sophisme : «Ou ces statues sont liées à des croyances idolâtres, ou il ne s'agit que de simples cailloux. Dans le premier cas, l'islam commande de les détruire; dans le second, qu'importe qu'on les brise. » On pourrait inverser le dilemme du Mullah, c'est un peu ce qu'a tenté l'ambassadeur : ou les statues de Bamiyan sont l'objet d'un culte, comme le sont celles des temples hindous de Kaboul, ou elles ne le sont pas. S'il y a des bouddhistes en Afghanistan, ils ont droit à la même tolérance que les hindous et les sikhs ; s'il n'y a en a pas, les statues ne peuvent être considérées comme des objets de culte, et qu'importe donc leur présence.

L'iconoclasme des talibans a été rattaché à plusieurs causes. Tout d'abord, des décisions de principe, essentielles, internes à la doctrine des talibans. Elles reposent sur la notion de souillure, d'une part, et sur une méfiance profonde pour la notion de patrimoine comme valeur "laïque ", d'autre part. Ensuite, il peut être expliqué par des causes externes, ou contextuelles sur lesquelles je reviendrai brièvement.

26 À la délégation pakistanaise venue, elle aussi, plaider la cause des bouddhas, Mullah Omar répliqua: "Comment pourrions justifier, au moment du Jugement dernier, d'avoir laissé subsister des impuretés sur le sol afghan?» D'où l'étonnant sacrifice de 
cent vaches ordonné par le Mullah. Il s'agit en réalité d'un double sacrifice: celui, purificateur, visant à l'élimination d'une souillure, c'est-à-dire des statues-idoles, sur l'autel du monothéisme, et celui, d'expiation, visant à effacer leur trop longue présence sur sol musulman.

La notion de patrimoine culturel hors de toute référence religieuse est suspecte aux yeux du Mullah. Il se méfie du patrimoine comme valeur laïque, et il n'a pas tort d'affirmer, dans son second décret, que "certains croient en ces statues». Certes, on peut, comme l'ont fait des commentateurs dans nos médias, reprocher au Mullah de confondre l'idole - illégitime donc pour le monothéisme - et l'icône, qui ouvre le regard et l'esprit vers l'invisible. Pour Mullah Omar, la délectation esthétique pourrait bien être une idolâtrie, ou du moins une occasion de distraire les hommes et les femmes de la lutte dans la voie de Dieu. Est condamnable donc, dans la perspective du Mullah, toute vénération, tout intérêt pour l'objet d'art qui impliquerait "une relation " religieusement non orthodoxe, c'est-à-dire esthétique, sensuelle, à l'invisible. Un objet, un monument, à la fois vénéré et détaché du sacré légitime est suspect. Pas de légitimité pour cet espace de respect et d'admiration, entre le culte dû à Dieu et les nécessités de la vie quotidienne, un espace où entreraient entre autres le passé préislamique d'Afghanistan, la délectation esthétique et un attachement identitaire qui ne repose pas sur la seule appartenance à l'oumma ${ }^{10}$.

En détruisant les bouddhas, les talibans se sont opposés à tout ancrage nationaliste, historique ou patrimonial dans le passé préislamique. On comprend que la population hazara ait ressenti la destruction comme une atteinte aux racines de son identité. Iconoclasme, visibilité et publicité sont intimement liés. Il fallait que l'acte définitif soit montré à la vue de tous, ou du moins qu'il soit visible au loin, qu'il ait des témoins, d'où l'autorisation de filmer la destruction donnée à Al-Jazira, qui en a diffusé des images reprises dans le monde entier, d'où également l'insistance à faire constater par des journalistes la vacuité du musée de Kaboul débarrassé de ses sculptures grécobouddhiques, et de transporter ces mêmes journalistes par avion quatre jours plus tard, à Bamiyan, où ils purent contempler les tas de gravats au pied des immenses niches désormais vides. Cette mise à mort appelait la présence d'observateurs aussi bien infidèles que croyants. Tout iconophobes qu'ils fussent, les talibans avaient donc besoin de l'image pour attester la destruction des images. Le Mullah a fait appel au témoignage par l'acte, comme le font les martyrs qui, en acceptant de mourir dans la voie de Dieu, témoignent à la fois de la vérité au nom de laquelle ils meurent et de l'ignominie de l'adversaire.

\section{Survie des bouddhas}

De quelle vérité l'anéantissement des bouddhas de Bamiyan témoigne-t-il ? Celle d'un monothéisme jaloux? Celle de la capacité des talibans d'être seuls contre tous, et de rester sourds aux appels à la sauvegarde, insensibles à l'émotion internationale ? La fumée de la pieuse pyrotechnie une fois dissipée, on vit qu'il n'y avait plus rien à voir que deux sarcophages vides.

Nous étions, ma femme et moi, à Phnom Penh, puis à Bangkok pendant les fatales journées de 2001. Dans ces pays bouddhistes, le choc fut plus profondément ressenti qu'en Occident. Il n'est pas exact que seul un Occident repu se soit ému de la destruction de ces statues de pierre et de stuc. Mais l'émoi ne fut pas le même. Je me 
souviens d'un débat dans les colonnes du Sunday Nation de Bangkok qui, comme le Cambodge Soir, consacrait des pages entières au sort des bouddhas de Bamiyan. Le Bouddha n'est pas dans ses statues, disaient les uns, il voulait qu'on s'attachât à son enseignement, pas à ses représentations matérielles. Pourtant, répliquaient les autres, les statues de Bouddha font partie de notre vie religieuse ; elles représentent un aspect essentiel de la vénération du Bouddha comme personnage à la fois historique et sacré. Elles ont, comme objet d'art et de culture, un lien matériel avec la doctrine du Bouddha, qui, paradoxalement, trouve sa vérité ultime dans le néant, concluaient les premiers.

31 Les médias, en Europe et aux États-Unis, se sont interrogés sur les causes contextuelles de la décision prise par Mullah Omar ; une question reprise dans le film tout récent de Christian Frei, The Giant Buddhas, qui confronte divers points de vue. Frei cite, par la bouche d'un cameraman d'Al-Jazira, un cinéaste iranien affirmant, mais l'argument a été répété souvent, que l'assaut contre les bouddhas serait une réaction à l'abandon dans lequel l'Occident aurait laissé l'Afghanistan, ou une manière de représailles contre les sanctions votées par le conseil de sécurité, et contre la non-reconnaissance de l'émirat. Après coup, des représentants des talibans ont exprimé « leur indignation et leur colère devant les protestations des pays "chrétiens" et leur souci exclusif pour des "idoles", contrastant avec [leur] insensibilité face aux malheurs des Afghans ».

32 Le patrimoine afghan, si les propos attribués aux talibans sont exacts, est ici placé en position d'otage, voire de victime indirecte de l'égoïsme des Occidentaux, plus émus par la disparition des statues de Bamiyan que par la misère de la population afghane. Mullah Omar impose ainsi à notre conscience une sorte de dilemme entre valeurs humanitaires et valeurs patrimoniales. Comme on sait, dans la réalité, le choix se présente rarement sous la forme de celui du bien contre le beau; plus souvent, et en Afghanistan même, la destruction du patrimoine s'ajoute aux malheurs quotidiens.

La destruction des bouddhas afghans suscita une émotion favorable à l'exhibition d'autres bouddhas dans le monde, brusquement sortis des réserves ou de l'oubli, tel ce bouddha dormant, de 14 mètres de long, découvert au Tadjikistan en 1966, oublié dans un dépôt, découpé en morceaux pour les besoins du stockage, et dont les archéologues de Douchanbe rassemblèrent les fragments de toute urgence pour l'exposer au musée de la capitale en août 2001, ou tel le bouddha démesuré de Leshan, dans la province de Sichuan, haut de 71 mètres, seize de plus que le grand bouddha de Bamiyan, dont on commençait la restauration dès avril 2001. Le bouddha de Leshan a la chance, lui, d'être officiellement inscrit au patrimoine mondial de l'UNESCO.

Enfin, il y a le projet de reconstitution du grand bouddha de Bamiyan à Leshan également, dans un parc à thèmes, une sorte de Buddhaland, mais il n'a pas encore été montré au public, à cause des protestations suscitées par l'emplacement choisi, qui abrite de nombreuses tombes de la dynastie des Han, déjà endommagées par les travaux de construction. Le phénomène de redécouverte, de reconstruction, de production de clones des bouddhas ou du Bouddha n'est pas sans faire penser à une nouvelle occurrence du Grand Miracle, la multiplication magique du Bouddha dans la tradition du tantrisme.

D'autres projets de reconstruction conjuguent le souci de conjurer une perte incommensurable et de ranimer le tourisme afghan. Dès avril 2001, on songea à reconstruire les Bouddhas. Un consortium fut constitué en Suisse dans cette intention : la destruction des tours du World Trade Center le 11 septembre 2001 et celle des bouddhas de Bamiyan ont quelque chose en commun, disait-on. Ce sont deux symboles 
du génie humain détruit par le terrorisme. On formula le projet d'une reconstruction in situ du Grand bouddha en béton spécial, à partir d'un relevé photogrammétrique fait dans les années soixante-dix; le coût en était évalué entre quarante et soixante millions de dollars. Dans une première étape, on réaliserait un bouddha virtuel en trois dimensions, dans un cyberspace où figurerait une exacte réplique de la statue. Dans une seconde étape, on la construirait à l'échelle d'un dixième pour étudier la résistance des matériaux. Dans la dernière, on reconstruirait le Grand bouddha lui-même, grandeur nature, sur place, ou à côté, dans une sorte de disneyland archéologique pour touristes à Bamiyan, laissant vide la niche du Petit bouddha comme témoin de l'acte barbare.

Le gouvernement afghan, consulté, est tenté par ce signe fort d'une reconstruction effaçant le décret des talibans. L'Unesco qui en 2003 se décide enfin à inscrire le site au patrimoine mondial, hésite; le coût est énorme, et les musulmans orthodoxes pourraient être choqués que l'on reconstruise des statues. Plus le temps passe, plus l'idée d'une reconstruction à l'identique apparaît irréaliste, voire irrévérencieuse. Le responsable du patrimoine à l'Unesco parle maintenant de tricherie et de profanation, et fait l'éloge du vide. Commençons, dit-il, par nous occuper de sauvegarder et de restaurer ce qui peut l'être, au musée de Kaboul et sur les sites archéologiques du pays. Il est décidé de consolider la falaise, ébranlée par les explosions, de préserver ce qui reste des fresques peintes des grottes. Pour ce faire, on expulse les habitants de ces cavités pour les héberger dans un nouveau village que l'on tarde hélas à construire. Enfin, on recueille minutieusement les moindres fragments, les moindres cailloux formant les tas de déblais au pied des niches, en vue d'une éventuelle reconstitution partielle.

Reconstruire les bouddhas à Bamiyan, n'est-ce pas un nouvel avatar de l'iconoclasme, celui qui prétend effacer par une reconstruction-reconstitution l'acte destructeur et ses traces? Pour celui qui fait aujourd'hui le voyage de Bamiyan, les immenses niches vides laissent une impression profonde. Mémoire, violence humaine, les silhouettes des bouddhas hantent encore la vallée ; les reconstruire à l'identique, dans leurs alvéoles, aboutirait à nier la négation que représente leur anéantissement. Ce dernier, après tout, déborde peut-être l'acte destructeur. La notion de patrimoine était jusqu'alors sans grande signification pour les Afghans aux yeux desquels comptaient surtout le passé et les monuments islamiques. Mais le patrimoine préislamique, associé à la mémoire de la fureur talibane, dont le sort a suscité tant d'émotion dans le monde, est désormais nommé, exprimé. Menacé de mort, amputé de ses fleurons les plus visibles, il est maintenant reconnu et considéré comme sien par un nombre croissant d'habitants de l'Afghanistan.

\section{NOTES}

1. 1. Maurice Fouchet, Notes sur l'Afghanistan. CEuvre posthume. Paris, Éditions Maisonneuve, 1931, 228 p., p. 125-126.

2. 2. Georges Le Fèvre, La Croisière jaune. Paris, Librairie Plon, 1933, XX-368 p., p. 51. 
3. 3. William Moorcroft and George Trebeck, Travels in the Himalayan Provinces of Hindustan and the Panjab, in Ladakh, Kashmir, in Peshawar, Kabul, Kunduz and Bokhara. 2 vol., New Delhi, Sagar Publications, 1971 (repr.), 450 p. et 508 p.

4. 4. Alexander Burnes, Travels into Bokhara. 3 vol., Londres, John Murray, 1835, 328 p., 384 p. et 304 p..

5. 5. Charles Masson: Narrative of various Journeys in Balochistan, Afghanistan, and the Panja, 3 vol., Londres, Richard Bentley, 1842, 472 p., 464 p. et 496 p., vol. II, p. 392-393.

6. 6. André Godard, Yeda Godard et Joseph Hackin, Les Antiquités bouddhiques de Bâmiyân. MDAFA

II. Paris et Bruxelles, Van Oest, 1928, 113 p. et ill., pl. VIII.

7. 7. Robert Byron, Route d'Oxiane. Paris, quai Voltaire, 1990, 381 p., p. 357.

8. 8. Coran, LIII, 19-20.

9. 9. Pierre Lafrance, «Comment les bouddhas de Bamyan n'ont pas été sauvés ». Critique internationale, $\mathrm{n}^{\circ} 12$, juillet 2001, p. 14-21.

10. 10. Voir aussi Jean-Michel Frodon, "The War of Images, or the Bâmiyân Paradox ", Bruno Latour et Peter Weibel (dir.), Iconoclash. Karlsruhe, Cambridge, Mass., Londres, MIT Press, 2002, 703 p., p. 221-223.

\section{RÉSUMÉS}

En mars 2001, on s'en souvient, à la suite d'un décret du Mullah Omar, les talibans procédèrent à la destruction, à coups d'explosifs, des deux Bouddhas géants de la falaise de Bamiyan en Afghanistan. Les démarches, vaines, tentées par l'UNESCO, par diverses organisations et par plusieurs gouvernements pour dissuader les talibans d'accomplir l'acte iconoclaste, puis l'émotion presque universelle, pays musulmans y compris, exprimée devant ce qui fut considéré comme un sacrilège, ou du moins comme un acte de barbarie, firent ressortir, autant que le deuil d'une œuvre appartenant au patrimoine de l'humanité, une confrontation entre des valeurs apparemment inconciliables: condamnation des images taillées et de l'idolâtrie, d'une part, préservation, voire « culte » même de ce que l'Occident, et pas seulement l'Occident, considérait comme un bien commun, universel, de l'autre. Dans cet article, on aborde, par delà les événements factuels, les enjeux et les valeurs exprimés dans l'argumentation des parties en cause : les bouddhistes, la population afghane et le public occidental.

March 2001-everyone remembers the event-the Taliban dynamited the two monumental Buddhas carved into the cliff face at Bamyan in Afghanistan, following a decree of Mullah Mohammed Omar. UNESCO, various other organisations and several governments tried in vain to prevent this iconoclastic act. The almost universal emotion that followed the destruction of the sculptures, including in many Islamic countries, saw the act as a sacrilegious one, or at least an act of barbarity, and revealed not only mourning for the loss of works belonging to world heritage, but also a confrontation between apparently irreconcilable values: on the one hand the condemnation of carved images and of idolatry, and, on the other, the preservation, the 'cult' even, in Western countries and elsewhere, for what is considered as a common cultural property of universal significance. This article, going beyond the simple facts of the case, examines the issues raised and the arguments expounded by the different actors: Buddhists, the population of Afghanistan and Western public opinion. 
Im März 2001 beschlossen die Taliban infolge eines Edikts des Chefs Mullah Omar die Sprengung der zwei riesigen in den Fels gehauenen Buddhasstatuen in Bamiyan. Alle von der UNESCO, von verschiedenen Organisationen und mehreren Regierungen angestellten Versuche, die Taliban von dieser schändlichen Tat abzuhalten, scheiterten. Diese Zerstörung rief sofort eine besonders heftige Empörung bei der internationalen Gemeinschaft hervor, selbst bei den islamischen Ländern. Sie wurde also von allen Seiten als ein Akt der Barbarei oder eine frevelhafte Schandtat verurteilt. Es ging nicht nur um den unersetzlichen Verlust eines Weltkulturerbes, sondern um die Auseinandersetzung zwischen zwei offensichtlich gegensätzlichen Wertvorstellungen: einerseits die Verurteilung der Bildhauereien und der Götzenverehrung, andererseits den Willen zum Schutz eines Kulturgutes, das vom Westen, aber nicht nur von ihm, als ein gemeinsames universelles Erbe betrachtet wurde. Über den auf Tatsachen beruhenden Inhalt hinaus werden in diesem Aufsatz die Zielsetzungen und die Werte der grundverschiedenen Argumentationen der Betroffenen abgewägt, nämlich der Buddhisten, der afghanischen Bevölkerung und des westlichen Publikums.

\section{AUTEUR}

\section{PIERRE CENTLIVRES}

Pierre Centlivres est né en 1933, docteur ès lettres, il est professeur émérite de l'Université de Neuchâtel (Suisse). Il a mené ses recherches en Afghanistan (1964-2005), au Pakistan (1986-1998) et en Suisse. Il a été conseiller au musée national afghan de Kaboul en1964-1966. Après avoir été conservateur-adjoint au département d'ethnographie du musée d'histoire de Berne (1967-1974) et chargé de cours au séminaire d'ethnologie de l'université de Berne (1971-1980), il succède en 1974 à Jean Gabus à la tête de l'Institut d'ethnologie de l'Université de Neuchâtel et fonde le centre de recherches ethnologiques (1974-1998). Il a crée la collection « Recherches et travaux » de l'Institut d'ethnologie, Neuchâtel et Paris, Maison des sciences de l'Homme, ainsi que la série «Ethnoscope ».

Ses principaux ouvrages comprennent sa thèse (Un bazar d'Asie centrale. Forme et organisation du bazar de Tâshqurghân, Afghanistan, Wiesbaden, Dr. L. Reichert, 1972), Chroniques afghanes 1965-1993 (Paris et Amsterdam, éditions des archives contemporaines, 1998), Les Bouddhas d'Afghanistan (Lausanne, éditions Favre, 2001), A seconde vue. Repères et perspectives en anthropologie (Gillion, Infolio, 2001). Avec Micheline Centlivres-Demont, il a signé Et si on parlait de l'Afghanistan? Terrains et textes, 1964-1980 (Neuchâtel, éditions de l'Institut d'ethnologie et Paris, MSH, 1988), Imageries populaires en Islam (Genève, Georg, 1997), Portraits d'Afghanistan (Paris, Adam Biro, 2002) et Revoir Kaboul. Chemins d'été, chemins d'hiver entre l'Oxus et l'Indus (Genève éditions Zoé, 2007). Sous sa direction ont été publiés : Une seconde nature. Pluralisme, naturalisation et identité en suisse Romande et au Tessin (avec Micheline Centlivres-Demont, Nadja Maillart et Laurence Ossipow, Lausanne, L'Age d'Homme, 1991), La Fabrique des héros (avec Daniel Fabre et Françoise Zonabend, Paris, MSH, 1998), Les Défis migratoires (avec Isabelle Girod, Zürich, éditions Seismo, 2000), Saints, sainteté et martyre. La fabrication de l'exemplarité (Neuchâtel, Institut d'ethnologie et Paris, MSH, 2001). 